\title{
The $H_{\text {I }}$ content of the advanced merger NGC 4441
}

\author{
E. Manthey ${ }^{1,2}$, S. Aalto ${ }^{3}$, S. Hüttemeister ${ }^{1}$, and T. A. Oosterloo ${ }^{2,4}$
}

1 University of Bochum, Department of Astronomy, 44780 Bochum, Germany
2 Astron, 7990AA Dwingeloo, The Netherlands
e-mail: manthey@astron. nl
3 Onsala Space Observatory, Chalmers University of Technology, 43992 Onsala, Sweden
4 Kapteyn Astronomical Institute, University of Groningen, PO Box 800, 9700AV Groningen, The Netherlands

Received 2 April 2007 / Accepted 3 April 2008

\section{ABSTRACT}

\begin{abstract}
Context. NGC 4441 is a candidate for a merger between a spiral and an elliptical galaxy (S+E merger), because it shows typical tidal structures such as an optical tail and two shells. With a far-infrared luminosity of $\sim 5 \times 10^{9} L_{\odot}$ this galaxy belongs to the class of moderate luminosity mergers, in which the merging process induces (if at all) only a moderate starburst.

Aims. The study of the atomic gas content allows us to investigate the merger history and the impact on the star formation. In particular, it is not clear from simulations whether an S+E merger leads to a gas concentration in the nucleus, resulting in a starburst, or whether the gas is spread out and therefore too diffuse for new star forming regions.

Methods. We used the Westerbork Radio Synthesis Telescope to observe the properties of the HI. By using this interferometer, we are able to study the large-scale H I distribution and kinematics with high spatial and velocity resolution.

Results. We found two H I tails extending out to more than $40 \mathrm{kpc}$. In a central disc, the gas shows a fairly regular rotation pattern indicating that the gas started to settle after the merger. The total H I mass adds up to $1.5 \times 10^{9} M_{\odot}$. By comparing the high resolution H I maps with deep optical images, differences between the stellar and gaseous tidal features are apparent, which indicates an $\mathrm{S}+\mathrm{E}$ merger origin.
\end{abstract}

Key words. galaxies: interactions - galaxies: starburst - radio lines: galaxies - radio lines: ISM

\section{Introduction}

Extreme mergers between two large disc galaxies, leading to a super-starburst and to ULIRGs (Sanders \& Mirabel 1996) have been at the focus of research, but those galaxies are rare. Small spirals and ellipticals dominate the galaxy population in many environments, but mergers between them, so-called $\mathrm{S}+\mathrm{E}$ mergers, have been poorly studied so far. Models of $\mathrm{S}+\mathrm{E}$ mergers are in strong disagreement concerning the prediction of enhanced star formation induced by the merger. Weil \& Hernquist (1993) used a smoothed particle hydrodynamics approach (SPH) to model the gas. With this fluid description, the stellar and gaseous components are quickly segregated. While the stars form features like shells, the gas congregates in the centre of the remnant galaxy, leading to a strong gas concentration and thus resulting in a starburst, similar to ULIRGs. Simulations by Kojima \& Noguchi (1997) however predict a dispersion of the gas clouds which might not lead to a starburst at all, because the density of the gas is too low to collapse and form new stars. They model the gas component as a system of inelastic cloud particles dissipating kinetic energy in mutual collisions. If the timescale for cloud-cloud collisions is relatively long, the cloud system behaves like a collisionless system, thus like the stellar component. Therefore, the gas and stellar distribution are similar. While the contrast of the structures is somewhat higher for the stellar component, both show shell-like features, as were also found for the stars in the models of Weil \& Hernquist (1993).

Simulations by Naab \& Burkert (2000), Naab \& Burkert (2001), Khochfar \& Burkert (2003) and Naab et al. (2006) revealed the importance of $\mathrm{S}+\mathrm{E}$ mergers for the formation of bright ellipticals. As Khochfar \& Burkert (2003) claim, these aspects are, however, poorly studied so far, and detailed observational investigations are needed besides more numerical simulations to better understand the process of those mergers.

One "prototypical" S+E merger candidate is NGC 4194, the Medusa. In the optical we see a diffuse tail going to the north and on the opposite side 2 shells are visible, as predicted by models. Molecular gas is found in a continuous distribution out to $4.7 \mathrm{kpc}$ from the centre, i.e. much more spread out than in the case of a ULIRG (Aalto \& Hüttemeister 2000; Aalto et al. 2001). However, this galaxy is clearly undergoing an intense starburst phase, albeit not as strong as in ULIRGs (Weistrop et al. 2004).

Here we present high resolution interferometric $\mathrm{HI}$ data of another S+E merger candidate, NGC 4441. This galaxy is morphologically very similar to the Medusa. It possesses one optical tidal tail and two bluish shells on the opposite side, which are, however, brighter, i.e. more evolved, than the shells in the Medusa. The main body has an elliptical shape (Bergvall 1981) with a small dust layer through the centre on the minor axis. Based on spectroscopic data Bergvall (1981) claims that this galaxy is a merger where most of the gas has been already used for star formation, which means only little ongoing star formation. However, there must have been a period of enhanced star formation, since the stellar population is younger than that of a normal elliptical galaxy (Bergvall 1981). Our own optical spectra confirm this and we estimate that a moderate starburst occured $\sim 1$ Gyr ago (Manthey et al. 2005).

The observations presented here were obtained with the Westerbork Synthesis Radio Telescope. We refer to the analysis 
Table 1. Basic properties of NGC 4441. The distance is based on $H_{0}=$ $75 \mathrm{~km} \mathrm{~s}^{-1} \mathrm{Mpc}^{-1}$.

\begin{tabular}{lc}
\hline \hline Property & \\
\hline RA $(2000)$ & $12: 27: 20.3$ \\
Dec $(2000)$ & $+64: 48: 05$ \\
$v_{\text {opt,hel }}\left(\mathrm{km} \mathrm{s}^{-1}\right)$ & 2722 \\
$D(\mathrm{Mpc})$ & 36 \\
type & $\mathrm{SAB} 0+\mathrm{pec}$ \\
$L_{\mathrm{B}}\left(10^{9} L_{\odot}\right)$ & 10.1 \\
$L_{\mathrm{FIR}}\left(10^{9} L_{\odot}\right)$ & 5.4 \\
$1^{\prime}$ & $10.5 \mathrm{kpc}$ \\
\hline
\end{tabular}

Table 2. Parameters for the WSRT observations.

\begin{tabular}{lc}
\hline \hline Observing parameters & \\
\hline Date & 28.2 .2003 \\
Centre frequency & 1408.9 \\
Total bandwidth & $20 \mathrm{MHz}$ \\
Number of channels & 1024 \\
Velocity resolution $\left(\mathrm{km} \mathrm{s}^{-1}\right)$ & 4.12 \\
Rms noise level (robust $=0.5)\left(\mathrm{mJy} \mathrm{beam}^{-1}\right)$ & 0.4 \\
Beamwidth (") & $20 \times 19$ \\
Primary beam & $0.6^{\circ}$ \\
Primary calibrator & $3 \mathrm{C} 48$ \\
\hline
\end{tabular}

of optical long-slit spectra for metalicity estimations, but these data are presented in detail in Manthey et al. (2008c).

In Table 1 general information on NGC 4441 is given. In Sect. 2 we give basic information about the observations obtained, in Sect. 3 we describe the data reduction for the interferometric data. Results are presented in Sect. 4, including derived properties like the $\mathrm{H}$ I gas mass and the star formation rate based on the $20 \mathrm{~cm}$ continuum flux. In Sect. 5 we discuss the observations with particular emphasis on models of mergers between Ellipticals and Spirals and we compare NGC 4441 with the S+E merger prototype NGC 4194 (the Medusa). Finally, in Sect. 6 we summarise the observations and results.

\section{Observations}

\subsection{WSRT}

The HI observations with the Westerbork Synthesis Radio Telescope (WSRT) were carried out in February 2003. Table 2 summarises the most important observing parameters. We observed NGC 4441 in one $12 \mathrm{~h}$ run in the maxi-short array configuration. This is best suitable for observations of an extended source within a single $12 \mathrm{~h}$ track. The quality of the data is good, no interference problems occurred. For bandpass and flux calibration, the calibrator 3C 48 was observed before the observations. Note that no secondary phase calibrator was observed. Instead, phase calibration at the WSRT is usually done using self-calibration. The chosen setup with a total bandwidth of $20 \mathrm{MHz}$ (corresponding to $\sim 4000 \mathrm{~km} \mathrm{~s}^{-1}$ ) and a velocity resolution of $4 \mathrm{~km} \mathrm{~s}^{-1}$ channel $^{-1}$ was capable to detect both narrow features in velocity space and a broad total velocity range due to tidal disruption. With the chosen setup, two polarisations were observed, with 1024 channels available for each of them.

\subsection{Optical imaging}

We present here an optical $R$ band image which was taken with CAFOS at the $2.2 \mathrm{~m}$ telescope on Calar Alto, Spain (CAHA) in
April 2004. To study very faint morphological features, we obtained deep imaging with an integration time of $60 \mathrm{~min}$. Standard reduction using IRAF was applied to the data. Here we use the optical image to compare the stellar and gaseous distribution. For further analysis of the optical data, we refer to Manthey et al. (2008c).

\section{Data reduction}

The HI data were reduced using MIRIAD $^{1}$ with some additional tasks written by $\mathrm{T}$. Oosterloo which are specific to the Westerbork interferometer ${ }^{2}$. After inspecting and, where necessary, flagging the calibrators, the WSRT data have to be calibrated first for changes in the system temperature. Since no secondary calibrator was observed, after flux and bandpass calibration a continuum image was created to perform selfcalibration on continuum sources within the field. To achieve a proper calibration, it is necessary to deeply CLEAN the continuum image. In an iterative process, first a mask was created which included only real emission to define the CLEANing components. Second, the image was CLEANed using the mask and subsequently a new, deeper mask could be generated for a better CLEANing. Once the image was finally CLEANed, selfcalibration was performed, and the loop started again with creating a mask, CLEANing and self-calibration. The data quality was high enough that only 3 iterations were necessary for a proper calibration. The results were then applied to the target galaxy. After continuum subtraction, a datacube was created to which we applied Hanning smoothing, leading to a velocity resolution of $8 \mathrm{~km} \mathrm{~s}^{-1}$. The datacube was convolved with a Gaussian of roughly double the size of the original beam. With this smoothed image a mask was created including real emission only. This was checked for all channels using the KARMA movie option (Gooch 1996). This mask was used for CLEANing as described above for the self-calibration process of the continuum. This CLEANing procedure was repeated 3 times until no significant sidelobes were visible. Integrated intensity and velocity maps were created using the MOMENT task in MIRIAD. Finally, primary beam correction was applied to the integrated intensity maps. We created maps which were naturally weighted, thus providing the highest sensitivity, as well as maps with a robust weighting of 0.5 to reach a higher spatial resolution. We reached an rms of $0.4 \mathrm{mJy}_{\text {beam }}^{-1}$ in the naturally weighted map and and rms of $0.5 \mathrm{mJy}^{\text {beam }}{ }^{-1}$ in the robustly weighted map. Here we present only the robust map with the higher spatial resolution, because the naturally weighted maps, although more sensitive, do not show any additional significant flux contribution compared to the robust map.

\section{Results}

\subsection{Neutral hydrogen}

\subsubsection{Morphology of the tidal tails}

The neutral hydrogen distribution in NGC 4441 shows two prominent tidal tails to the north and to the south (see Fig. 1). The southern arm is blueshifted with respect to the systemic velocity (see Fig. 2), while the northern arm has a higher velocity. Both tails have a similar extent: the northern tail out to $48 \mathrm{kpc}$, the southern tail out to $42 \mathrm{kpc}$ from the nucleus and both form

\footnotetext{
1 http://www.atnf.csiro.au/computing/software/miriad/

2 http://www . astron.nl/ oosterlo/wsrtMiriad/
} 

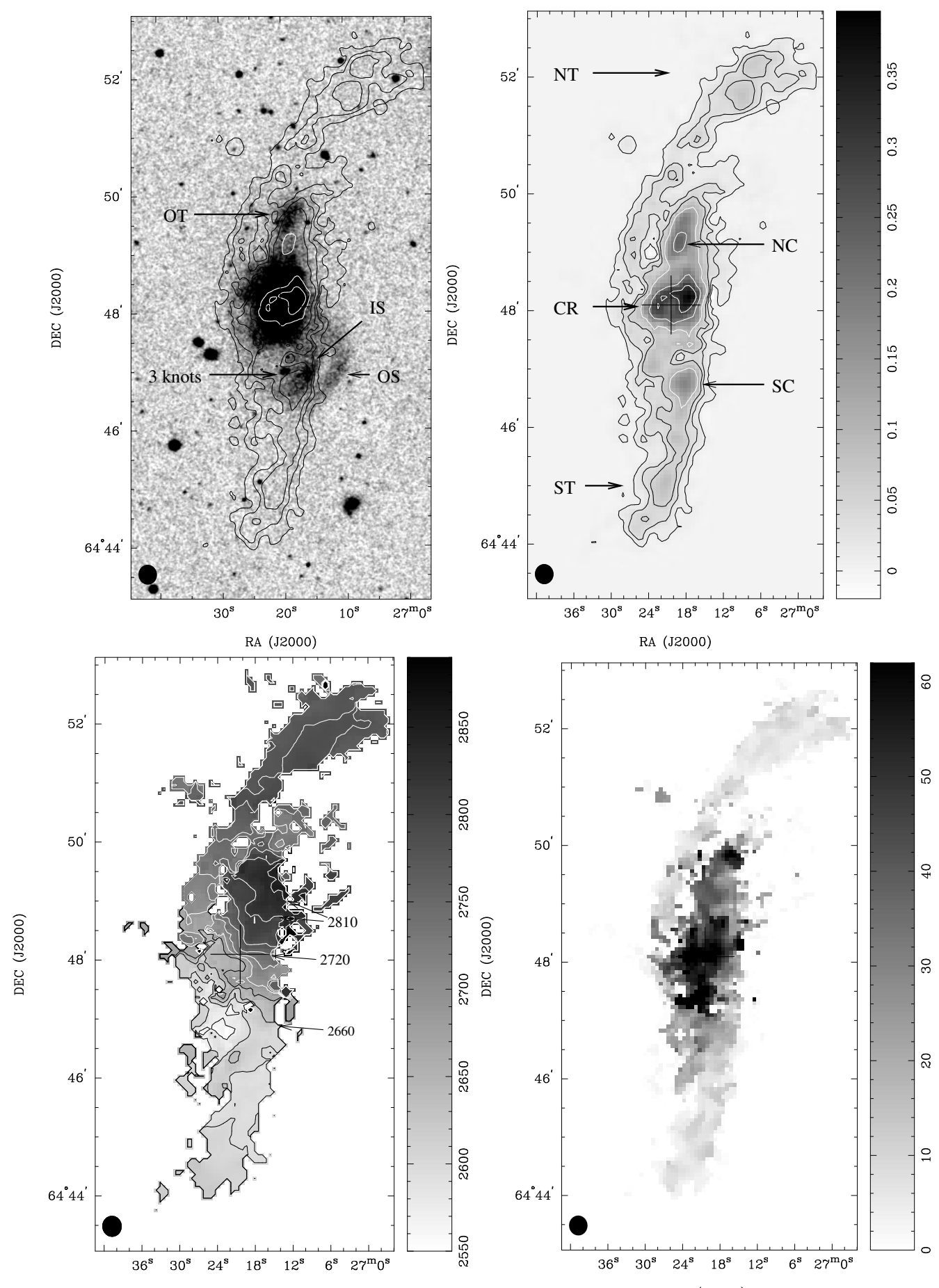

Fig. 1. NGC 4441 - top left: H I distribution overlayed on optical DSS image. Contour levels are 2.8, 8.4, 14, 28, 56, $84 \times 10^{19} \mathrm{~cm}^{-2} \mathrm{Marked}$ are the optical features: the optical tail (OT), the inner shell (IS), the outer shell (OS) and the three optical knots, top right: H I distribution, contours same as above. Marked are the gaseous features as described in the text: the northern tail (NT), the northern clump (NC), the central ring (CR), the southern clump (SC), and the southern tail (ST), bottom left: velocity field, contour levels are 2570 to $2840 \mathrm{~km} \mathrm{~s}^{-1}$, in steps of $30 \mathrm{~km} \mathrm{~s}^{-1}$, bottom right: H I velocity dispersion (2nd moment). The cross marks the optical centre position.

a relatively symmetric structure. The H I tails are clumpy, including the tip of both tails. Two large clumps are found, both $\sim 17 \mathrm{kpc}$ from the centre (marked as NC and SC in Fig. 1). From the kinematics there are no indications that these clumps are or will be separate gravitationally bound entities like those simulated by Duc \& Bournaud (2007).

It is noticeable that the symmetry in the $\mathrm{HI}$ distribution is not seen in the optical morphology. Here, there is a tail to the north which coincides nicely in position and extension with the large northern H I clump. In the southern direction, however, we see a misalignment of the $\mathrm{HI}$ tail and the optical tidal features. Two shells are visible in the optical southwest of the main body. There is almost no overlap of the more western, outer shell with the H I tail. The tip of the inner shell corresponds to the southern H I clump. Three optical knots are found at that position, of which at least two do not look point-like, i.e., are probably not 


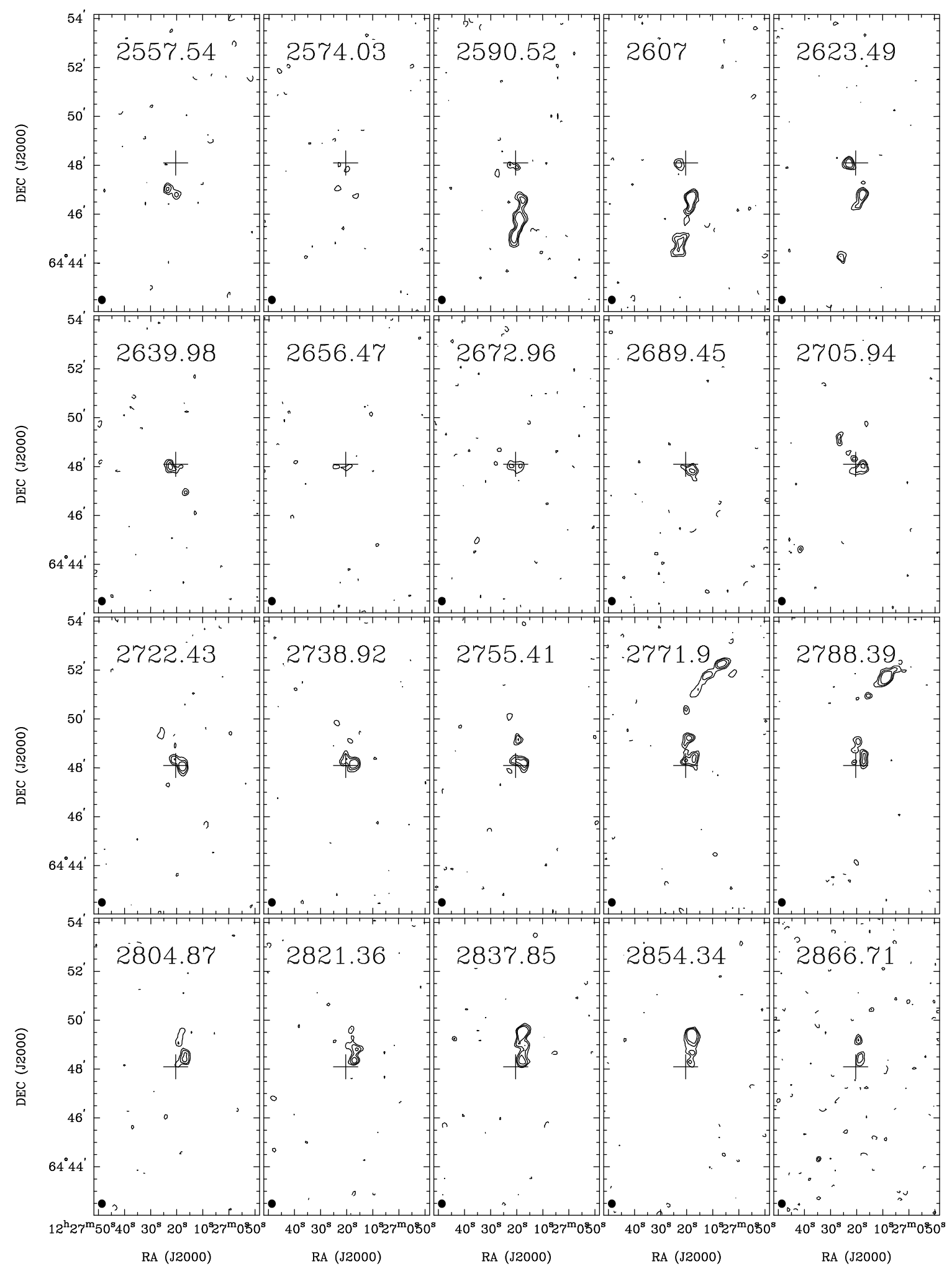

Fig. 2. Channel maps (robust weighted) of NGC 4441. The optical centre position is marked with a cross. The contour levels are $-0.9,1.1,1.3$, $1.5,1.7,1.8,2 \mathrm{mJy}$ beam $^{-1}$. The heliocentric velocity is given at the top left, the beam at the bottom left corner.

foreground stars. They might be star forming knots, probably too close to the galaxy to survive as tidal dwarf galaxies, but can also be background galaxies, because our deep $R$-band image shows a background galaxy cluster. However, no H I is detected at higher velocities within our band, which may of course miss distant galaxies. Unfortunately, the distance of the background cluster is not known.

The gas in the tails shows only little dispersion in general $\left(<15 \mathrm{~km} \mathrm{~s}^{-1}\right)$. The dispersion increases slightly in regions of gradients in the atomic gas distribution, e.g., around clumps. 
Table 3. The H I properties of the total system, the tails and the central disc of NGC 4441. Heliocentric velocities are given.

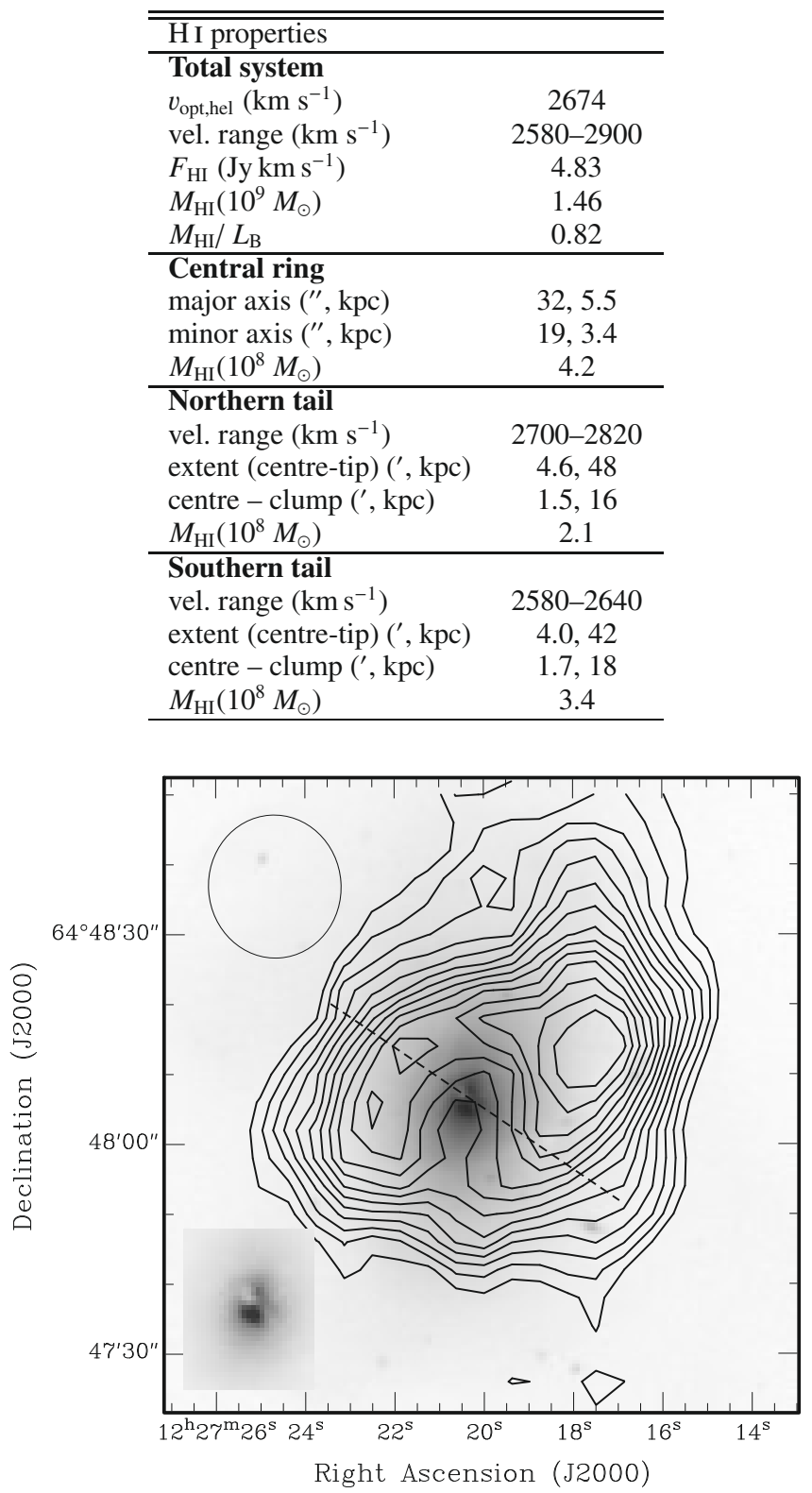

Fig. 3. $R$-band image (taken with the CAHA $2.2 \mathrm{~m}$ telescope) of the central region. $\mathrm{HI}$ is overlayed as contours, contour levels are from 2.8 to $10.08 \times 10^{20} \mathrm{~cm}^{-2}$ in steps of $0.56 \times 10^{20} \mathrm{~cm}^{-2}$. The beamsize is shown in the upper left corner. The gas ring is centred on the optical nucleus, which exhibits a thin dust lane. The orientation of the dust lane is marked by the dashed line. In the lower left corner, the $R$-band image of the nucleus with the dust lane is shown.

\subsubsection{The central ring}

Figure 3 shows the central region of NGC 4441 in the optical $R$ band and the $\mathrm{HI}$ distribution (robust $=0.5$ ) overlayed as contours. We find a distinct $\mathrm{H}$ I ring with a diameter of $\sim 11 \mathrm{kpc}$ centred on the optical (and CO) nucleus. At the centre of the ring there is an HI depression, which is, however, unresolved. The highest concentration of $\mathrm{H}$ I is found on the west side of the ring, thus, west of the optical centre. Three H I peaks are present in the ring, with a distance of $\sim 2.5 \mathrm{kpc}$ from the centre. This ring structure might actually be a small disc, where in the inner part
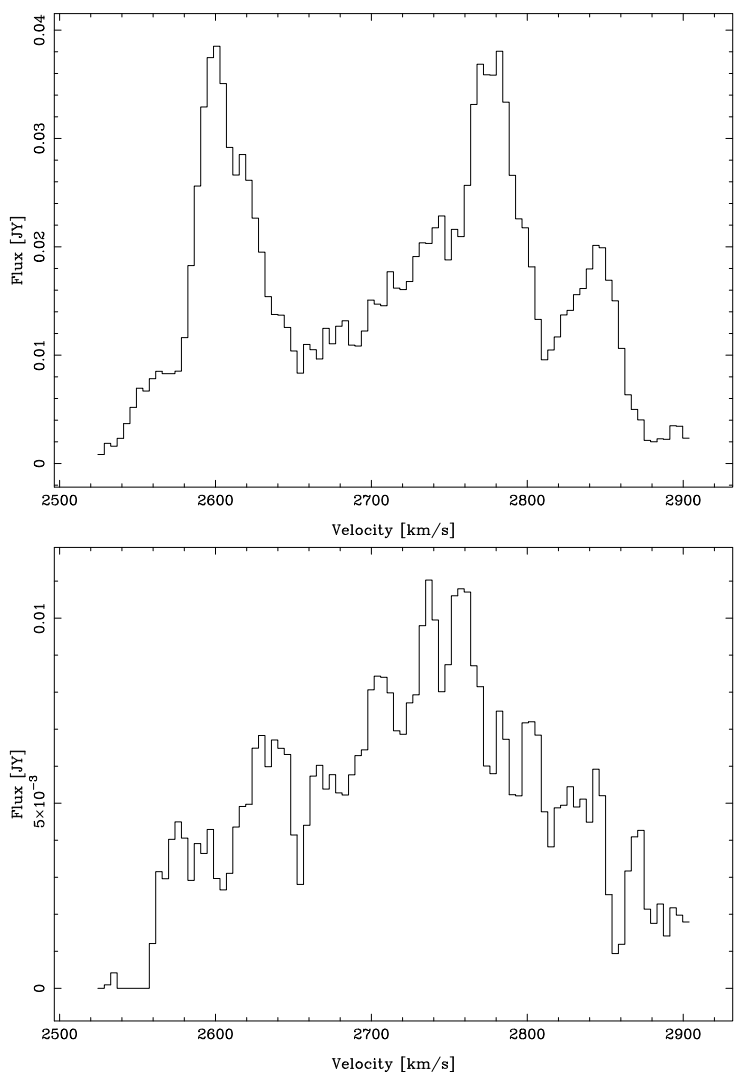

Fig. 4. Integrated HI spectrum of top: the total amount of $\mathrm{HI}_{\mathrm{I}}$ in NGC 4441 and bottom: the central ring. In the total H I spectrum, the two narrow features around $2600 \mathrm{~km} \mathrm{~s}^{-1}$ and $2780 \mathrm{~km} \mathrm{~s}^{-1}$ correspond to the two tidal tails. The third peak at $2840 \mathrm{~km} \mathrm{~s}^{-1}$ appears at the position of the northern clump.

most of the gas is present in the molecular phase which is often found at column densities of $\sim 10^{21} \mathrm{~cm}^{-2}$ (Young 2000).

Figure 4 shows the integrated H I spectra of the total H I amount and the central ring. The total H I spectrum shows three peaks. The features around $2600 \mathrm{~km} \mathrm{~s}^{-1}$ and $2780 \mathrm{~km} \mathrm{~s}^{-1}$ correspond to the southern and northern tidal tail. A third peak at $2840 \mathrm{~km} \mathrm{~s}^{-1}$ appears at the position of the northern clump (see also Fig. 2).

\subsubsection{Kinematics}

The main body of the H I distribution shows a relatively regular rotation pattern which allows at least a crude estimate of the dynamical mass. Note that this region is much larger than the central ring seen in the $\mathrm{H}$ I distribution map and has a different position angle.

In Fig. 5 the region of the central velocity field is shown. Using the axis ratio it is possible to estimate geometrically the inclination. Even though drawing an ellipse is approximate, it is not arbitrary here. Based on the elliptical shape of the velocity field shown in Fig. 5 it is clear the the disc is seen relatively edge-on, thus the uncertainty of the inclination does not affect the mass calculation significantly.

We measured an axis ratio of $\sim 1 / 3$, equivalent to an inclination of $\sim 70^{\circ}$. An error of $\pm 20^{\circ}$ leads to an uncertainty of a factor of 2 in mass and thus gives at least an indication of the mass range. Note, that the minor axis is however not perpendicular to the major axis, which is a hint for non-radial motions like infall or outflow that could be triggered by the merger. One possible 


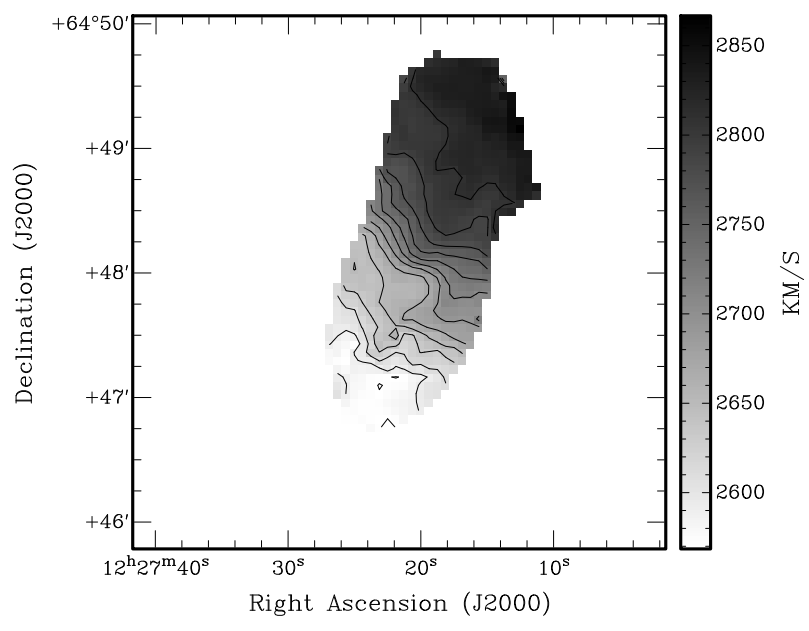

Fig. 5. Inner part of velocity field of NGC 4441, which shows a fairly regular solid body rotation pattern. The contour levels are from 2775 to $2825 \mathrm{~km} \mathrm{~s}^{-1}$, in steps of $25 \mathrm{~km} \mathrm{~s}^{-1}$.

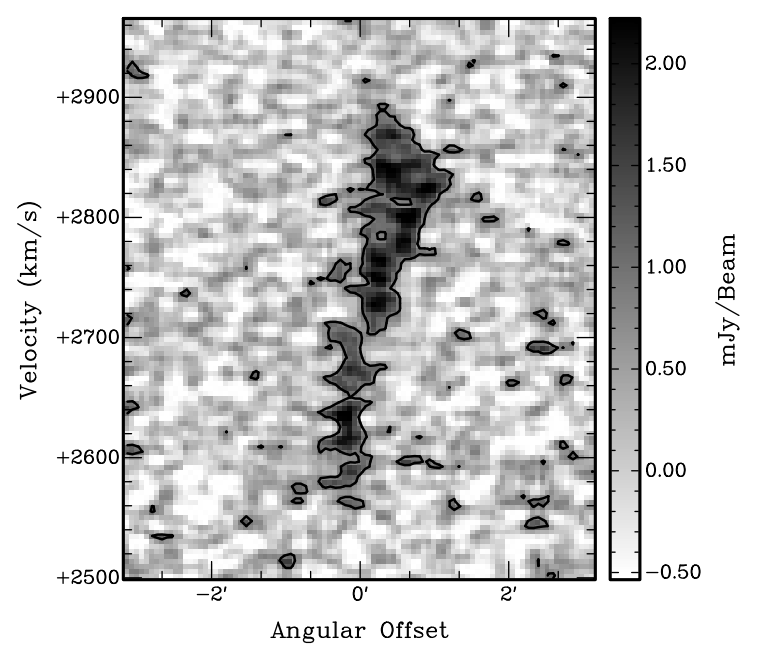

Fig. 6. Position-velocity diagram of the major axis of the inner $\mathrm{H}$ I disc in NGC 4441. The position angle is $325^{\circ}$. Shown are the $3 \sigma$ contours of $0.9 \mathrm{mJy}$ beam $^{-1}$.

explanation would be an elongated gravitational potential, e.g., a bar or spiral arms, which forces the gas to move on ellipsoidal orbits. The resolution is, however, not sufficient to investigate this in more detail. Furthermore, the minor axis corresponds to the thin dust lane seen in the central region (see Fig. 3).

In Fig. 6 we present the position-velocity-diagram (pvdiagram) through the major axis of the H I disc (position angle $(\mathrm{pa})=325^{\circ}$ ). It shows a broad velocity range which is expected for a highly inclined disc. In the centre there is a gap because of the local minimum in the H I distribution. A second velocity component at high velocities appears in the inner part, which demonstrates the complexity of the velocity distribution in this merger remnant.

With an inclination of $\sim 70^{\circ}$ and a maximum $\mathrm{H}$ I rotation velocity of $\sim 163 \mathrm{~km} \mathrm{~s}^{-1}$ at a distance of $1^{\prime}$ (estimated from the pv-diagram), we derive a dynamical mass of $\sim 6 \times 10^{10} M_{\odot}$ (see Table 4). Even though this is a crude estimate, the mass-to-light ratio is within the range of what is found in normal galaxies.
Table 4. Estimated parameters of the central H I disc in NGC 4441.

\begin{tabular}{lc}
\hline \hline Estimated parameters & \\
Disc extent (") & 60 \\
Max. velocity $\left(\mathrm{km} \mathrm{s}^{-1}\right)$ & 163 \\
Position angle $\left(^{\circ}\right)$ & -35 \\
Inclination $\left(^{\circ}\right)$ & 70 \\
\hline$M_{\mathrm{dyn}}\left(10^{10} M_{\odot}\right)$ & 6.4 \\
$M_{\mathrm{HI}} / M_{\mathrm{dyn}}$ & 0.028 \\
$M_{\mathrm{dyn}} / L_{\mathrm{B}}\left(M_{\odot} / L_{\mathrm{B}, \odot}\right)$ & 6.3 \\
\hline
\end{tabular}

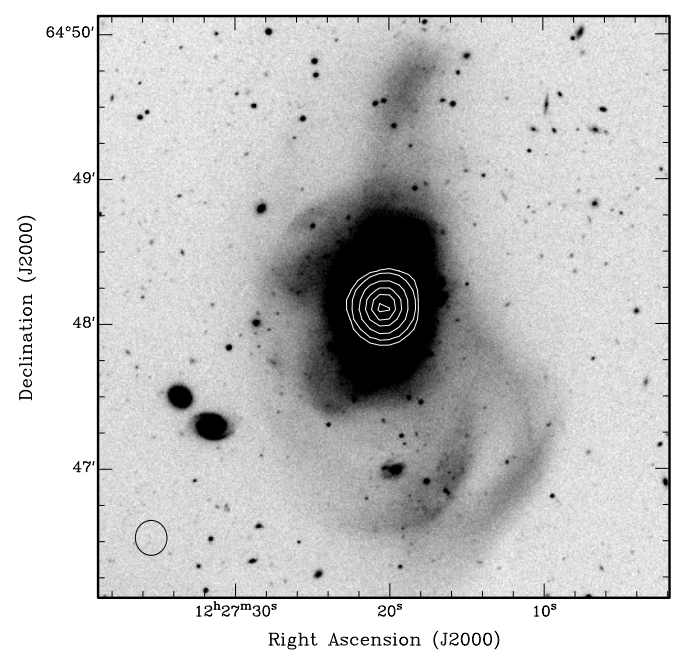

Fig. 7. $R$-band image (CAHA) and contours of the $20 \mathrm{~cm}$ continuum flux. The beam is shown in the lower left corner of the image. Contour levels are $0.5,1,2,5,7.5,10 \mathrm{mJy}_{\text {beam }}{ }^{-1}$.

\section{2. $20 \mathrm{~cm}$ continuum}

Figure 7 shows an optical image with the $20 \mathrm{~cm}$ radio continuum overlaid as contours. The continuum source in NGC 4441 is barely resolved and concentrated on the nucleus of the galaxy. We fitted a Gaussian to the continuum source using the MIRIAD task IMFIT and derived a deconvolved source size of less than $1 \mathrm{kpc}$. The derived centre coordinates corresponds to the optical coordinates. To estimate the star formation rate (SFR) from the 20 -cm radio continuum flux density we use

$\operatorname{SFR}\left(M_{\odot} \mathrm{yr}^{-1}\right)=0.14 D^{2} F_{20 \mathrm{~cm}}$

taken from Condon (1992) and Haarsma et al. (2000), where $D$ is the distance in $\mathrm{Mpc}$ and $F_{20 \mathrm{~cm}}$ is the $20-\mathrm{cm}$ radio continuum flux density in Jy. In Table 5 the results for NGC 4441 are given. Table 5 also gives the SFR estimated from the FIR luminosity:

$\operatorname{SFR}\left(M_{\odot} \mathrm{yr}^{-1}\right)=0.17 L_{\mathrm{FIR}}$

following Kennicutt (1998), with $L_{\mathrm{FIR}}$ in units of $10^{9} L_{\odot}$. The IRAS $60 \mu \mathrm{m}$ and $100 \mu \mathrm{m}$ flux densities were taken from Moshir (1990). The $S F R$ of $1-2 M_{\odot} \mathrm{yr}^{-1}$ calculated with both methods is low for a merger and normal for typical spirals.

We also calculated the logarithmic ratio of FIR to radio flux, the so-called $q$ parameter (Helou et al. 1985):

$q=\log \frac{F(\text { FIR }) /\left(3.75 \times 10^{12} \mathrm{~Hz}\right)}{F(1.4 \mathrm{GHz})}$

Note, that the continuum flux at $1.4 \mathrm{GHz}, F(1.4 \mathrm{GHz})$ in this formula, is in units of $\mathrm{W} \mathrm{m}^{2} \mathrm{~Hz}^{-1}=10^{26} \mathrm{Jy}$. The FIR flux, $F($ FIR), is a combination of the $60 \mu \mathrm{m}$ and $100 \mu \mathrm{m}$ flux densities, as used 
Table 5. Continuum and FIR fluxes, SFR based on continuum and FIR emission, respectively, and $q$ parameter.

\begin{tabular}{lc}
\hline \hline Key characteristics & \\
\hline $20 \mathrm{~cm}(\mathrm{Jy})$ & 0.013 \\
$60 \mu \mathrm{m}(\mathrm{Jy})$ & 2.7 \\
$100 \mu \mathrm{m}(\mathrm{Jy})$ & 3.9 \\
$S F R_{20} \mathrm{~cm}\left(M_{\odot} \mathrm{yr}^{-1}\right)$ & 2.4 \\
$S F R_{\mathrm{FIR}}\left(M_{\odot} \mathrm{yr}^{-1}\right)$ & 1.0 \\
$q$ & 2.4 \\
\hline
\end{tabular}

for determining $L_{\mathrm{FIR}}: F(\mathrm{FIR})=1.26 \times 10^{-14} \cdot(2.58 \cdot F(60 \mu \mathrm{m})+$ $F(100 \mu \mathrm{m})$ ). To calculate $q, F(\mathrm{FIR})$ is normalised by the mean frequency of $60 \mu \mathrm{m}$ and $100 \mu \mathrm{m}$, which is $3.75 \times 10^{12} \mathrm{~Hz}$. Helou et al. (1985) derived a mean value for $q$ of 2.3. Although this parameter was determined for disc galaxies only (but independent of their star formation activity), NGC $4441(q=2.4)$ conforms to this value.

\section{Discussion}

\subsection{The merger origin}

For an understanding of the whole merger process of galaxies resulting in a remnant like NGC 4441, it is necessary to know the progenitors. Of course it is difficult, if not impossible, to get a unique answer to that question from a completely merged remnant. However, there are some indications from our observations which at least narrow the parameter space.

Since we found a large amount of gas in NGC 4441, at least one spiral galaxy must be involved in the merger process. In the following, we discuss the possibility of a spiral + spiral $(S+S)$ and spiral+elliptical $(\mathrm{S}+\mathrm{E})$ merger.

\section{Spiral+elliptical merger?}

There are several arguments that support an $\mathrm{S}+\mathrm{E}$ merger origin:

No strongly enhanced star formation: typically, $\mathrm{S}+\mathrm{S}$ merger remnants which show strong tidal features comparable to what is seen in NGC 4441, host a region of a (fading) starburst, e.g., Hibbard \& van Gorkom (1996). By looking at the FIR luminosity, the $20 \mathrm{~cm}$ continuum emission and the optical spectra (Manthey et al. 2005), however we do not find any indication for a strong starburst, neither ongoing nor in the recent history, in NGC 4441. Therefore, less gas is probably involved in this merger event, which is naturally the case when one progenitor is an elliptical.

It has to be mentioned, however, that the strength of an induced starburst not only depends on the available amount of gas but also on the internal structure of the progenitor galaxies. In particular, the presence of a bulge in a progenitor spiral galaxy will stabilise the gas disc against the formation of a bar during the merger (Mihos 1999a). That bar would be a powerful mechanism to support infall of gas into the central region leading to a strong starburst (Mihos \& Hernquist 1994). In S+S merger simulations gas dissipates energy and thus loses angular momentum when it becomes shocked along a bar (Barnes \& Hernquist 1991, 1996; Mihos \& Hernquist 1996; Barnes 1999). This leads to rapid gas inflows and a concentration of gas in the centre of the remnant, resulting in a starburst.

Nevertheless, the simulations for $\mathrm{S}+\mathrm{S}$ with bulges, i.e., no merger-induced bars, never show such a low star formation rate as in the case of NGC 4441.
Of course, it is necessary to investigate in numerical simulations how bar formation and a present bulge influences the gas in a $S+E$ merger scenario, in particular when the galaxies are completely merged. Due to the spheroidal shape of the elliptical which is similar to a bulge, bar formation is likely not important and therefore no strong gas inflows occur. Since the major and minor axes of NGC 4441 are not perpendicular, this might indicate the presence of a small oval distortion which is, however, not strong enough to trigger enhanced star formation.

Theoretical models: various numerical simulations showed that optical shells in combination with tidal tails are typical for S+E merger remnants (Quinn 1984; Dupraz \& Combes 1986; Hernquist \& Quinn 1988; Kojima \& Noguchi 1997; Combes \& Charmandaris 1999). These shells are created by phase wrapping of the disc in the potential well of the elliptical galaxy (Quinn 1984). They occur in the advanced merger stage $\sim 4-5 \times 10^{8} \mathrm{yr}$ after the impact and last for more than 1 Gyr (Quinn 1984; Kojima \& Noguchi 1997).

The simulated gas distribution strongly depends on the model used for the ISM. It has been noted by Combes \& Charmandaris (1999) and Charmandaris \& Combes (2000) that the often used smoothed particle hydrodynamics (SPH) approach might not always be appropriate. Instead, these authors account for the clumpy structure of the ISM by simulating the behaviour of individual gas clouds rather than a continuous fluid. They found a largely spread gas distribution, often forming gaseous tails as we see in NGC 4441. Therefore, these models do not predict a strong starburst phase, because the gas becomes too dispersed. This agrees with our observations, but stands in contrast to S+S merger scenarios (e.g., Barnes \& Hernquist 1991; Mihos 1999b).

Stellar component similar as the Medusa: the similarity of the optical morphologies of NGC 4441 and NGC 4194, the prototypical S+E merger remnant, has been mentioned in the introduction, reflecting a possible analogue merger origin. Besides the obvious features like the stellar shells and tidal tails, both galaxies host a dust lane.

In NGC 4441, the minor axis of the fairly regularly rotating $\mathrm{HI}$ disc corresponds to a faint central dust lane (Fig. 3). It is interesting to note that also the Medusa exhibits a minor axis dust lane. Although this may further strengthen the notion of a a similar origin, the dust lane of the Medusa is more prominent and longer (it curves into the optical tail). Furthermore, that dust lane is associated with a double spectral feature (Aalto \& Hüttemeister 2000). Such extra features are not seen in NGC 4441. This may be a result of its more evolved state, because a large fraction of the ISM has been consumed in star formation.

H I vs. optical morphology: in NGC 4441 we see striking differences between the stellar and gaseous distribution. In particular the two optical shells stand in contrast to the two symmetric $\mathrm{H}$ I tails.

In $\mathrm{S}+\mathrm{S}$ merger remnants, however, the optical and H I morphology are generally similar (Hibbard \& van Gorkom 1996), because both the stellar and gaseous tidal features are formed from the two discs which are merging. Hibbard et al. (2000) found some examples in which the optical and the gaseous tails are displaced or where some regions are lacking gas, while the overall morphologies are still similar. The authors described several scenarios for these discrepancies between the stellar and gaseous tidal morphology and claim that mainly superwinds are responsible for the differences found. These superwinds are driven by the central starburst occuring in $\mathrm{S}+\mathrm{S}$ merger remnants and sweep away the gas in the direction of the wind. 
Their findings differ, however, in two ways from NGC 4441. First, NGC 4441 does not host an ongoing strong starburst, neither did it in the recent history. Therefore, the existence of a superwind is unlikely. Second, we see not only an offset of the gaseous and stellar tidal features, but also a completely different morphology. This cannot be explained by a possible superwind alone. Other possible explanations given by Hibbard et al. (2000), like dust-obscuration and photoionisation, can only explain local differences and not large-scale discrepancies, as pointed out by the authors. Photoionisation might explain why there is only little gas found in the outer optical shell. In that case we expect to find a large amount of ionised gas which can be detected in e.g. H $\alpha$ emission. This will be done in a further investigation.

\section{Spiral+Spiral merger?}

There is one striking difference between NGC 4441 and $\mathrm{S}+\mathrm{E}$ merger simulations as well as the prototype NGC 4194:

Two symmetric H I tails: the discussion of the merger origin of N GC 4441 is complicated by the finding of two symmetric $\mathrm{HI}$ tails. This is typical for a merger between two disc galaxies, when each disc forms one tail during the encounter (e.g., Toomre \& Toomre 1972; Hernquist \& Barnes 1994; Mihos \& Hernquist 1996). Neither the simulations by Weil \& Hernquist (1993) nor Kojima \& Noguchi (1997) show the appearance of two tails in an $\mathrm{S}+\mathrm{E}$ merger scenario. Furthermore, our H I data of the prototypical S+E merger NGC 4194 show only one gaseous tail in that galaxy, in accordance to the $\mathrm{S}+\mathrm{E}$ merger simulations (Manthey et al. 2008b).

Observations of the interacting S+E galaxy system Arp 140 (an early stage of an $\mathrm{S}+\mathrm{E}$ merger where both galaxies are still clearly separated) done by Cullen et al. (2007) showed as well one Hi tail only. Furthermore, they found an Hi bridge between the interacting galaxies formed by disc material which streams towards the elliptical galaxy (Toomre \& Toomre 1972). This bridge will likely be destroyed once the galaxies finally merge, as in the case of bridges found in $\mathrm{S}+\mathrm{S}$ mergers (Toomre \& Toomre 1972; Hibbard 1995).

In recent deep observations of early-type galaxies Morganti et al. (2006) found a significant amount of $\mathrm{H} \mathrm{I}$ in a large fraction of those galaxies. The data reveal a variety of structures, including extended discs. However, the peak column density is with $N(\mathrm{HI}) \leq 10^{20} \mathrm{~cm}^{-2}$ lower than what we found in NGC 4441 $\left(N(\mathrm{HI})=1.1 \times 10^{21} \mathrm{~cm}^{-2}\right)$, so no star formation occurs in the regular early-type galaxies of the Morganti et al. (2006) sample. However, considering the likely $\mathrm{S}+\mathrm{E}$ merger origin of NGC 4441, it is possible that the elliptical progenitor possessed such a gas disc, which would explain why NGC 4441 has two $\mathrm{H}$ I tidal tails and otherwise resembles an S+E merger remnant.

The low column density $\mathrm{H} \mathrm{I}$ disc around ellipticals might also explain why the star formation in NGC 4441 is not as enhanced by the merger as in "normal" disc-disc mergers.

\subsection{The formation of the central HI disc}

Clearly, NGC 4441 is in an advanced merger stage, because even the progenitor nuclei are completely merged in addition to the pronounced stellar tidal features. In this stage a stable gas disc can already have formed, as shown by Barnes (2002). In unequal-mass mergers the disc formation is more impressive (Barnes 2001), which again supports that NGC 4441 is at least not an advanced remnant of a merger between two large disc galaxies.

The fairly regular rotation pattern in the central HI disc indicates that the merger event has happened several hundred million years ago (e.g., Mihos 1999a). The gas in this advanced merger has already started to settle down, but without reaching an equilibrium state yet. The lack of H I in the centre (Figs. 1, 6) might be caused by an efficient transformation of atomic to molecular gas. This is supported by our CO detection within the central 22" (Manthey et al. 2008a).

The velocity gradient of the central disc connects to that of the two tidal tails, even though perturbations are clearly present. That means that the material in the tails and the central disc share the same sense of rotation. This indicates that a large amount of angular momentum still remains in the gas of the tidal tails. A merger geometry in which the angular momentum does not cancel out much seems therefore likely, e.g., a prograde merger with parallel rotation spins of the galaxies. Independent of the presence of an $\mathrm{H} \mathrm{I}$ disc in the elliptical progenitor, a prograde merger is likely in which the rotation of the spiral and the movement of the spiral towards the elliptical are aligned.

The amount of conserved angular momentum in the gas also depends on other factors, e.g., the presence of a bar formed during the interaction in which the gas loses angular momentum by shocks (see above).

If no strong shocks occur, the gas retains much of its angular momentum, which prevents the gas from falling toward the central region on short timescales. Instead, a gaseous disc will be formed. The remaining gas in the tidal tails will finally return to the main body and will lead to the growth of the present H I disc. This behaviour is modelled by e.g., Barnes (2002) and is observed in other merger remnants like NGC 7252 (Hibbard et al. 1994; Hibbard \& Mihos 1995) and NGC 4038/39 (Hibbard et al. 2001).

\subsection{The star forming region}

NGC 4441 has a moderate ongoing star formation rate of 1-2 $M_{\odot} \mathrm{yr}^{-1}$. However, there are indications based on optical spectroscopy that there has been a period of enhanced star formation $\sim 1$ Gyr ago (Bergvall 1981; Manthey et al. 2005).

The extent of the star-forming region can be investigated using the continuum data. The deconvolved size of the $20 \mathrm{~cm}$ continuum source was determined as $<1 \mathrm{kpc}$ (Table 5). The emission comes likely from ongoing star formation only, since the $q$ value agrees with the FIR-to- $20 \mathrm{~cm}$ continuum ratio typical for star formation, and based on optical spectroscopy there are no hints for another origin, e.g. an AGN (Bergvall 1981). Manthey et al. (2008a) found molecular gas extended out to $\sim 4 \mathrm{kpc}$ in NGC 4441. Thus, the region of ongoing star formation is embedded in the reservoir of raw material for star formation, namely the molecular gas extent, but does not cover its full extent. Compared to the Medusa, where the region of ongoing star formation is extended on a scale of $\sim 2 \mathrm{kpc}$ (e.g., Armus et al. 1990) and much more intense $\left(S F R_{\mathrm{opt}} \sim 30 M_{\odot} \mathrm{yr}^{-1}\right)$, the star forming activity seems to be shrunk in intensity and maybe also in spatial extent. The question arises, why the starburst in NGC 4441 has already faded, while the Medusa is still intensely forming stars. Aalto \& Hüttemeister (2000) estimate that the burst in the Medusa will continue only for $\sim 40 \times 10^{6}$ years, under the assumption that the current star formation rate will not change dramatically. If the star formation rate depends mainly on the duration of the burst and decreases with ongoing burst age, the phase of intense star formation in a merger event, which 
NGC 4441 and the Medusa both underwent, might be very short, and the Medusa will fade soon as well.

Furthermore, it has become clear by simulations of $\mathrm{S}+\mathrm{S}$ mergers that the starburst intensity in mergers of unequal masses strongly depends on the structure of the progenitors (e.g., the presence of a bulge) and the geometry of the merger event (orbits, spin orientation of the galaxies etc.) (Cox et al. 2007). Even though no detailed simulations exist to investigate this phenomenon in the case of $\mathrm{S}+\mathrm{E}$ mergers, it is likely that these factors play a similar important role in $\mathrm{S}+\mathrm{E}$ mergers. Therefore, (unequal-mass) $\mathrm{S}+\mathrm{E}$ mergers are expected to show a much larger scatter in starburst strength even if they are in the same merger stage.

\section{Summary}

1. We observed the moderate luminosity merger NGC 4441 with the Westerbork radio interferometer and found a complex, extended H I structure affected by tidal forces. The total H I mass adds up to $1.46 \times 10^{9} M_{\odot}$.

2. We detected two H I tails. Both tails have a similar extent out to $4^{\prime}$ (42 kpc, southern tail) and 4.6' (48 kpc, northern tail) away from the centre and they emerge from opposite sides of the main galactic body.

3. The northern H I arm follows the optical tail closely, however the $\mathrm{HI}$ is more extended and shifted to the east. In contrast, the southern HI arm is not aligned with the shell structure found in the optical, but touches only the inner optical shell, whereas in the outer shell no neutral gas is found.

4. The velocity field shows a fairly regular rotation pattern in the inner region, associated with the optical main body. The overall velocity gradient is continued in the tidal tails. The southern tail has a generally lower velocity as the system, the velocity of the northern tail is larger than the central velocity.

5. From the $20 \mathrm{~cm}$ continuum flux we calculated the star formation rate as $2.4 M_{\odot} \mathrm{yr}^{-1}$. From the FIR fluxes we get a $S F R$ of $1.0 M_{\odot} \mathrm{yr}^{-1}$. The resulting ratio $q$ is 2.4 , which is in good agreement with the typical q value in the literature. We determined a continuum source size of less than $1 \mathrm{kpc}$.

6. Because of the large differences in the stellar and gaseous distribution, it is unlikely that NGC 4441 is a merger between two large disc galaxies. If differences are found in those mergers, they are on much smaller scales and can be best explained by superwinds (see Hibbard et al. 2000). In NGC 4441, no such strong galactic winds are expected, because the galaxy is in a phase of moderate star formation. A merger between two ellipticals can be also excluded, because we found a large amount of atomic and molecular gas and signs of a past strong star formation activity. This properties are not expected in $\mathrm{E}+\mathrm{E}$ mergers. Thus, it is most likely, that NGC 4441 is the remnant of an S+E merger.

Acknowledgements. The WSRT is operated by the Netherlands Foundation for Research in Astronomy (ASTRON), with support of the Netherlands Organisation for Scientific Research (NWO). This research has made use of the NASA/IPAC Extragalactic Database (NED) which is operated by the Jet Propulsion Laboratory, California Institute of Technology, under contract with the National Aeronautics and Space Administration. The research was supported by the German Science Organisation (DFG) through the Graduiertenkolleg 787.

\section{References}

Aalto, S., \& Hüttemeister, S. 2000, A\&A, 362, 42

Aalto, S., Hüttemeister, S., \& Polatidis, A. G. 2001, A\&A, 372, L29

Armus, L., Heckman, T. M., \& Miley, G. K. 1990, ApJ, 364, 471

Barnes, J. E. 1999, in Galaxy Dynamics - A Rutgers Symposium, ed. D. R. Merritt, M. Valluri, \& J. A. Sellwood, ASP Conf. Ser., 182, 463

Barnes, J. E. 2001, in Highlights of Spanish astrophysics II, ed. J. Zamorano, J. Gorgas, \& J. Gallego, 85

Barnes, J. E. 2002, MNRAS, 333, 481

Barnes, J. E., \& Hernquist, L. 1996, ApJ, 471, 115

Barnes, J. E., \& Hernquist, L. E. 1991, ApJ, 370, L65

Bergvall, N. 1981, A\&A, 97, 302

Charmandaris, V., \& Combes, F. 2000, in Small Galaxy Groups, ed. M. J. Valtonen, \& C. Flynn, ASP Conf. Ser. 209, IAU Colloq., 174, 273

Combes, F., \& Charmandaris, V. 1999, in Galaxy Dynamics - A Rutgers Symposium, ed. D. R. Merritt, M. Valluri, \& J. A. Sellwood, ASP Conf. Ser., 182,489

Condon, J. J. 1992, ARA\&A, 30, 575

Cox, T. J., Jonsson, P., Somerville, R. S., Primack, J. R., \& Dekel, A. 2007, ArXiv e-prints, 709

Cullen, H., Alexander, P., Green, D. A., \& Sheth, K. 2007, MNRAS, 376, 98

Duc, P.-A., \& Bournaud, F. 2007, ArXiv e-prints, 710

Dupraz, C., \& Combes, F. 1986, A\&A, 166, 53

Gooch, R. 1996, in Astronomical Data Analysis Software and Systems V, ed. G. H. Jacoby, \& J. Barnes, ASP Conf. Ser., 101, 80

Haarsma, D. B., Partridge, R. B., Windhorst, R. A., \& Richards, E. A. 2000, ApJ, 544,641

Helou, G., Soifer, B. T., \& Rowan-Robinson, M. 1985, ApJ, 298, L7

Hernquist, L., \& Barnes, J. E. 1994, in Mass-Transfer Induced Activity in Galaxies, ed. I. Shlosman, 323

Hernquist, L., \& Quinn, P. J. 1988, ApJ, 331, 682

Hibbard, J. E. 1995, Ph.D. Thesis, AA (Columbia University)

Hibbard, J. E., Guhathakurta, P., van Gorkom, J. H., \& Schweizer, F. 1994, AJ, 107,67

Hibbard, J. E., \& Mihos, J. C. 1995, AJ, 110, 140

Hibbard, J. E., Vacca, W. D., \& Yun, M. S. 2000, AJ, 119, 1130

Hibbard, J. E., van der Hulst, J. M., Barnes, J. E., \& Rich, R. M. 2001, AJ, 122 , 2969

Hibbard, J. E., \& van Gorkom, J. H. 1996, AJ, 111, 655

Kennicutt, R. C. 1998, ARA\&A, 36, 189

Khochfar, S., \& Burkert, A. 2003, ApJ, 597, L117

Kojima, M., \& Noguchi, M. 1997, ApJ, 481, 132

Manthey, E., Hüttemeister, S., Haberzettl, L., \& Aalto, S. 2005, in The Evolution of Starbursts, ed. S. Hüttmeister, E. Manthey, D. Bomans, \& K. Weis, AIP Conf. Proc., 783, 343

Manthey, E., Aalto, S., \& Hüttemeister, S. 2008a, A\&A, submitted

Manthey, E., Hüttemeister, S., Aalto, S., \& Horellou, C. 2008b, in prep.

Manthey, E., Jütte, M., Hüttemeister, S., \& Aalto, S. 2008c, in prep.

Mihos, C. 1999a, Ap\&SS, 266, 195

Mihos, J. C. 1999b, in Galaxy Interactions at Low and High Redshift, ed. J. E. Barnes, \& D. B. Sanders, IAU Symp., 186, 205

Mihos, J. C., \& Hernquist, L. 1994, ApJ, 425, L13

Mihos, J. C., \& Hernquist, L. 1996, ApJ, 464, 641

Morganti, R., de Zeeuw, P. T., Oosterloo, T. A., et al. 2006, MNRAS, 371, 157

Moshir, M. 1990, in IRAS Faint Source Catalogue, version 2.0, 0

Naab, T., \& Burkert, A. 2000, in Dynamics of Galaxies: from the Early Universe to the Present, ed. F. Combes, G. A. Mamon, \& V. Charmandaris, ASP Conf. Ser., 197, 267

Naab, T., \& Burkert, A. 2001, in Galaxy Disks and Disk Galaxies, ed. J. G. Funes, \& E. M. Corsini, ASP Conf. Ser., 230, 453

Naab, T., Jesseit, R., \& Burkert, A. 2006, MNRAS, 372, 839

Quinn, P. J. 1984, ApJ, 279, 596

Sanders, D. B., \& Mirabel, I. F. 1996, ARA\&A, 34, 749

Toomre, A., \& Toomre, J. 1972, ApJ, 178, 623

Weil, M. L., \& Hernquist, L. 1993, ApJ, 405, 142

Weistrop, D., Eggers, D., Hancock, M., et al. 2004, AJ, 127, 1360

Young, J. S. 2000, in Mapping the Hidden Universe: The Universe behind the Mily Way - The Universe in HI, ed. R. C. Kraan-Korteweg, P. A. Henning, \& H. Andernach, ASP Conf. Ser., 218, 321 